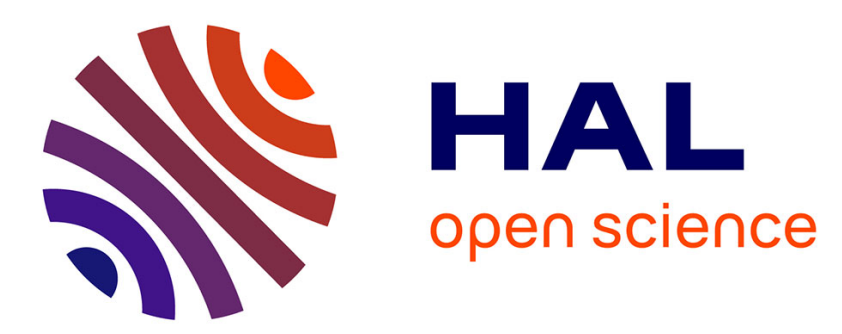

\title{
Effect of C5-Methylation of Cytosine on the UV-Induced Reactivity of Duplex DNA: Conformational and Electronic Factors
}

\author{
Akos Banyasz, Luciana Esposito, Thierry Douki, Marion Perron, Clément \\ Lepori, Roberto Improta, Dimitra Markovitsi
}

\section{To cite this version:}

Akos Banyasz, Luciana Esposito, Thierry Douki, Marion Perron, Clément Lepori, et al.. Effect of C5Methylation of Cytosine on the UV-Induced Reactivity of Duplex DNA: Conformational and Electronic Factors. Journal of Physical Chemistry B, 2016, 120, pp.4232-4242. 10.1021/acs.jpcb.6b03340 . cea01334656

\author{
HAL Id: cea-01334656 \\ https://hal-cea.archives-ouvertes.fr/cea-01334656
}

Submitted on 21 Jun 2016

HAL is a multi-disciplinary open access archive for the deposit and dissemination of scientific research documents, whether they are published or not. The documents may come from teaching and research institutions in France or abroad, or from public or private research centers.
L'archive ouverte pluridisciplinaire HAL, est destinée au dépôt et à la diffusion de documents scientifiques de niveau recherche, publiés ou non, émanant des établissements d'enseignement et de recherche français ou étrangers, des laboratoires publics ou privés. 


\section{Effect of C5-Methylation of Cytosine on the UV- induced Reactivity of Duplex DNA -}

\section{Conformational and Electronic Factors}

Akos Banyasz, ${ }^{a}$ Luciana Esposito, ${ }^{b}$ Thierry Douki, ${ }^{c}$ Marion Perron, ${ }^{a}$ Clément Lepori, ${ }^{a}$ Roberto Improta $^{* b}$ and Dimitra Markovitsi ${ }^{* a}$

${ }^{a}$ CNRS, IRAMIS, LIDYL, Laboratoire Francis Perrin, URA 2453, F-91191 Gif-sur-Yvette,

France

${ }^{\mathrm{b}}$ IstitutoBiostrutture e B

${ }^{c}$ Univ. Grenoble Alpes, INAC, LCIB, LAN \& CEA, INAC, SCIB, LAN, F-38000 Grenoble, France

AUTHOR INFORMATION

\section{Corresponding Authors}

thierry.douki@cea.fr

robimp@unina.it

dimitra.markovitsi@cea.fr 


\section{ABSTRACT}

C5-methylation of cytosines is strongly correlated with UV-induced mutations detected in skin cancers. Mutational hot-spots appearing at TCG sites are due to the formation of pyrimidine cyclobutane dimers (CPDs). The present study, performed for the model DNA duplex $(\text { TCGTA })_{3} \cdot(\text { TACGA })_{3}$ and the constitutive single strands, examines the factors underlying the effect of C5-methylation on pyrimidine dimerization at TCG sites. This effect is quantified for the first time by quantum yields $\phi$. They were determined following irradiation at 255, 267 and $282 \mathrm{~nm}$ and subsequent photoproduct analysis using HPLC coupled to mass spectrometry. C5methylation leads to an increase of the CPD quantum yield up to $80 \%$ with concomitant decrease of that of pyrimidine(6-4) pyrimidone adducts (64PPs) by at least a factor of three. The obtained $\phi$ values cannot be explained only by the change of the cytosine absorption spectrum upon C5methylation. The conformational and electronic factors that may affect the dimerization reaction are discussed in the light of results obtained by fluorescence spectroscopy, molecular dynamics simulations and quantum mechanical calculations. Thus, it appears that the presence of an extra methyl on cytosine affects the sugar puckering, thereby enhancing conformations of the TC step that are prone to CPD formation but less favorable to 64PPs. In addition, C5-methylation diminishes the amplitude of conformational motions in duplexes; in the resulting stiffer structure, $\pi \pi^{*}$ excitations may be transferred from initially populated exciton states to reactive pyrimidines giving rise to CPDs.

\section{KEYWORDS}

DNA methylation, quantum chemistry, cyclobutanes dimers, (6-4) photo-adducts, photochemistry, 


\section{INTRODUCTION}

Important biological functions, associated with gene expression and genome reprogramming, are regulated via methylation/demethylation of cytosines (C) at their 5 position. ${ }^{1-2}$ But 5 methylated cytosines $(5 \mathrm{mC})$ have been also correlated with carcinogenic mutations induced by UV radiation. This minor nucleobase is considered to be involved in about $30 \%$ of mutational hotspots detected in the p53 gene of skin tumors. ${ }^{3-4}$ Mutations were found in CCG/TCG sites (T and G stand, respectively, for thymine and guanine) and mutational events have been connected with the formation of cyclobutanepyrimidine dimers (CPDs).

Several studies on di- and tri-nucleotides, model duplexes, genomic DNA and cells found that the CPD yield increases upon C5-methylation. ${ }^{5-9}$ However, the reported reactivity enhancements are not comparable because no quantum yields are available and the conclusions depend on the specific experimental conditions used in each experiment. Another important discrepancy in the literature reports concerns the role of the irradiation wavelength. As a matter of fact, the increase in mutation frequency due to C5-methylation was found to be one order of magnitude higher for UVB irradiation compared to UVC. ${ }^{10}$ Therefore, it was attributed to the red shift of the absorption spectrum of $5 \mathrm{mC}$ with respect to that of $\mathrm{C}$ (Figure 1a). But a much smaller difference was determined between the yields of the UVB- and UVC-induced CPDs in isolated genomic DNA. $^{8}$ In addition, a difficulty preventing correct evaluation of the reactivity of methylated duplexes arises from deamination of T5mC CPDs (Scheme 1). This process, occurring in dark, transforms T5mC CPDs into TT CPDs. ${ }^{11-12}$ As a result, it is not possible to discriminate between CPDs arising from $\mathrm{T} 5 \mathrm{mC}$ and $\mathrm{TT}$ sites unless analysis at the nucleotide level in specific sequences is performed. 


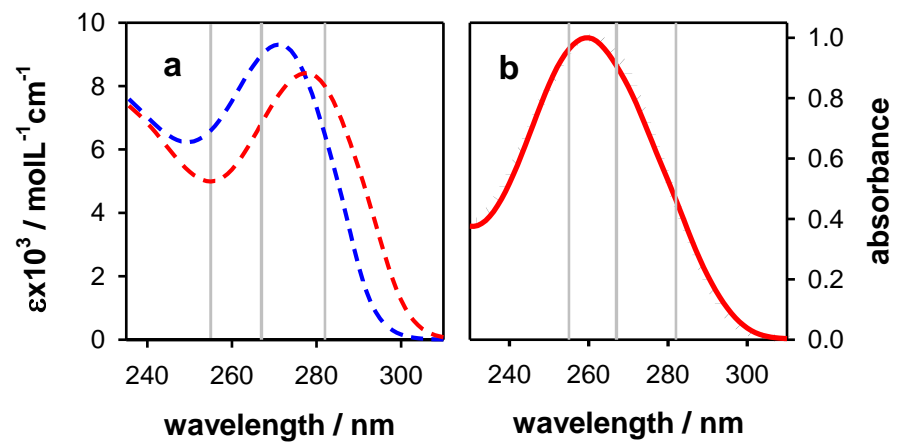

Figure 1. Comparison of the absorption spectra of (a) dC (blue) and 5mdC (red) and (b) the methylated duplex mds and the corresponding stoichiometric mixture of mono-nucleosides. Spectra in (b) are normalized to their maximum. Vertical grey lines denote the irradiation wavelengths.

In the light of the above considerations, it appears that the starting point for understanding the role of C5-methylation in the photoreactivity of duplex DNA is the determination of quantum yields. These quantum yields should correspond to dimerization reactions occurring exclusively in bipyrimidine sites that can be selectively methylated. This was precisely the first objective of the present study. It has been performed for duplexes with 15 base pairs containing the relevant sequence TCG in its non-methylated and methylated form: (TCGTA) $)_{3} \cdot(\mathrm{TACGA})_{3}$ and $(\text { T5mCGTA })_{3} \cdot(\text { TACGA })_{3}$, abbreviated as n-ds and m-ds, respectively. In such a base sequence, pyrimidine dimers originate solely from TCG sites. For comparison, we have also studied the corresponding single strands (TCGTA $)_{3}$ and (T5mCGTA $)_{3}$, abbreviated as n-ss and m-ss, respectively. We note that the complementary single strand (c-ss) (TACGA) ${ }_{3}$, common in n-ds and $\mathrm{m}$-ds, does not contain reactive bipyrimidine sites.

We determined quantum yields for pyrimidine dimerization, analyzing not only CPDs but also the second type of dimeric phototoproducts, pyrimidine(6-4) pyrimidone adducts (64PPs), whichso far attracted less attention than CPDs (Scheme 1). Irradiations were carried out at 255 
and $266 \mathrm{~nm}$ and $282 \mathrm{~nm}$. The photoproducts were quantified by HPLC coupled to mass spectrometry.

Scheme 1: Formation of $\mathrm{T} 5 \mathrm{mC}$ dimeric photoproducts. CPD: cyclobutane pyrimidine dimers, 64PP: pyrimidine (6-4) pyrimidone photoproducts, dR: 2-deoxyribose

The dimerization quantum yields determined for our model systems clearly show that the effect of C5-methylation on pyridine dimerization cannot be explained simply by the difference between the absorption spectra of $\mathrm{C}$ and $5 \mathrm{mC}$. Therefore, we explored other factors that may affect the dimerization reactions. Thus, we pursued our investigation using fluorescence spectroscopy, molecular dynamics simulations (MD) and quantum mechanical (QM) calculations.

Fluorescence spectroscopy provided information about the redistribution of the excitation energy within single and double strands. ${ }^{13}$ This is reflected in the way that C5-methylation affects the fluorescence quantum yields of the $\pi \pi^{*}$ emission and the low-energy emission bands due to charge transfer transitions. Then, molecular dynamics (MD) simulations, already used for the study of pyrimidine dimerization, ${ }^{9,14-15}$ allowed us to investigate the effect of C5-methylation on the conformational behavior of the studied systems and, in particular, on the most important 
structural parameters modulating the dimerization reactions. Finally, we evaluated the effect of C5-methylation on the Franck-Condon excited states, by performing QM calculations on representative fragments of the examined systems: on the one hand, DNA duplexes with three base pairs composed of the relevant sequences $(\mathrm{TCG})_{\mathrm{ds}}$ and $(\mathrm{T} 5 \mathrm{mCG})_{\mathrm{ds}}$; on the other, singlestranded pentamers $(\text { TCGTA })_{\mathrm{ss}} /(\mathrm{T} 5 \mathrm{mCGTA})_{\mathrm{ss}}$ and $(\mathrm{ATCGT})_{\mathrm{ss}} /(\mathrm{AT} 5 \mathrm{mCGT})_{\mathrm{ss}}$ in the duplex geometry, in order to assess the role of the bases flanking the central sequence.

The second part of our work, based on three complementary approaches, shows how the presence of an extra methyl at the TCG sequence may modify conformational and electronic parameters that play a key role in the UV-induced dimerization reactions. It highlights the complexity of the involved processes and sheds light on the subtle interplay of different chemical physical effects modulating the DNA photoreactivity.

\section{EXPERIMENTAL DETAILS}

Materials. DNA oligomers, HPLC purified, were obtained from Eurogentec Europe as single strands. Equimolar mixtures $\left(2 \times 10^{-4} \mathrm{molL}^{-1}\right)$ of complementary single strands were dissolved in

phosphate buffer $\left(0.1 \mathrm{molL}^{-1} \mathrm{NaH}_{2} \mathrm{PO}_{4}, 0.1 \mathrm{molL}^{-1} \mathrm{Na}_{2} \mathrm{HPO}_{4}, 0.25 \mathrm{molL}^{-1} \mathrm{NaCl}\right)$ prepared using Millipore water (Milli-Q Synthesis) and annealed in a dry heat bath (Eppendorf Thermostat Plus). To this end the solutions were successively (i) kept at $95^{\circ} \mathrm{C}$ for $15 \mathrm{~min}$, (ii) cooled to the estimated melting temperature of the duplex where they were maintained for $30 \mathrm{~min}$ and (iii) slowly cooled down to room-temperature. Typical melting curves are shown in Figure SI-1. The strand concentration in the experiments varied between $0.5 \times 10^{-6}$ and $2 \times 10^{-6} \mathrm{molL}^{-1}$.

Spectroscopic measurements. Steady-state absorption and fluorescence spectra were obtained using a Perkin Lambda 900 spectrophotometer and a SPEX (Fluorolog-3, Jobin-Yvon) spectrofluorimeter, respectively. Emission spectra were recorded at a right-angle configuration 
and were corrected for the response of the detection system. Temperature control was achieved by a Huber CC3 apparatus. Fluorescence quantum yields $\phi_{\mathrm{f}}$ were determined using TMP as a reference $\left(\phi_{\mathrm{f}}=1.54 \times 10^{-4}\right) .{ }^{16}$ In order to avoid artifacts related to the very weak fluorescence of nucleic acids, we applied specifically developed experimental protocols. ${ }^{17-18}$

Irradiations. Continuous irradiations were performed using the Xe lamp of the SPEX spectrofluorimeter with a monochromator bandwidth of $5 \mathrm{~nm}$. During the irradiation the temperature of the solution, which was mildly stirred, was kept at $23 \pm 0.1^{\circ} \mathrm{C}$. The intensity of the exciting beam was continuously monitored by a photodetector which was calibrated before and after the experiment by a powermeter (OPHIR/PD300-UVNIST traceable).

Photoproducts analysis. Oligomers were enzymatically hydrolyzed to release unmodified bases as nucleosides and photoproducts as dinucleoside monophosphates. Two 2-hour incubation periods were performed at $37^{\circ} \mathrm{C}$, first with phosphodiesterase II, DNase II and Nuclease P1 (pH 6), then with phosphodiesterase I and alkaline phosphatase ( $\mathrm{pH} 8)$. The obtained solutions were analyzed by HPLC-MS/MS using negative electrospray ionization. Selective quantification of the dimeric photoproducts was achieved by multiple reaction monitoring. ${ }^{7}$ In this detection mode, the first quadrupole of the mass spectrometer is set at the $\mathrm{m} / \mathrm{z}$ value of the targeted pseudo-molecular ion. Subsequently, these ions are directed into the second quadrupole, where they are fragmented by collision with molecular nitrogen. The resulting fragments are then directed into the third quadrupole that is set at $\mathrm{m} / \mathrm{z}$ values specific for the targeted compounds. For CPDs, both the cis,syn $(c, s)$ and trans,syn $(t, s)$ diastereoisomers were quantified. Fragmentations used for TC photoproducts have been reported previously. ${ }^{7}$ The $544 \rightarrow 195$ transition was used for the $t, s$ CPDs and 64PPs of T5mC. The $c, s$ T5mC CPD was quantified as itsdeaminated TT derivative due to quantitative deamination during enzymatic hydrolysis. In 
contrast, $t, s \mathrm{~T} 5 \mathrm{mC}$ CPDs were more stable; yet, $t, s$ TT CPD resulting from its deamination was also detected.

\section{COMPUTATIONAL DETAILS}

Molecular dynamics simulations. The single strands studied by MD simulations correspond to n-ss and m-ss, as defined in the introduction. In the case of duplexes, in order to avoid fraying, we considered longer structures (19-mers), containing additional GC sequences at both ends of the $5^{\prime} \rightarrow 3$ 'strand, and subsequently we analyzed the central 15 -mers, corresponding to n-ds and m-ds. As we have a repetitive sequence, we distinguished three different TCG/T5mCG triplets, numbered 1 to 3 , along the $5^{\prime} \rightarrow$ 3'direction. Each oligomer was constructed with a canonical BDNA conformation. A single very long (500 ns) MD simulation was performed for each system. Trajectories were obtained using state-of-art simulation conditions derived by the $\mathrm{ABC}$ consortium $^{19}$ and already successfully used in the study of TCG trimers. ${ }^{9}$ Indeed, MD simulations were done with periodic boundary conditions within a truncated octahedral cell, using the Amber11 suite of programs ${ }^{20}$ and the parmbs0 refinement ${ }^{21}$ to the parm99 force field. ${ }^{22-23}$ We used a SPC/E water model ${ }^{24}$ and $\mathrm{K}^{+}$ions to achieve neutrality; the ionic strength was set to about $0.15 \mathrm{molL}^{-1}$ by adding $\mathrm{KCl}$ salt. The structures were immersed in a truncated octahedral water box whose dimensions ensured a $11 \AA$ solvation shell around the structure. Electrostatic interactions were treated using the particle mesh Ewald method with a real space cut-off of $9 \AA$. Lennard-Jones interactions were truncated at $9 \AA$. The Berendsen algorithm was used to control temperature and pressure, with a coupling constant of 5 ps for both parameters. Parameters for the $5 \mathrm{mC}$ were derived from Lankas et al. ${ }^{25}$ The systems were equilibrated using previously published protocols. ${ }^{9,}, 19$ 
The trajectory structures were visually inspected using VMD $1.9 .^{26}$ The analysis of the parameters was conducted using the ptraj module of Amber11. The 3DNA program ${ }^{27}$ was used to analyze the simulations of single-stranded structures as well as to extract the vectors normal to the aromatic plane of the bases and the distances between the geometric centers of the bases. The Curves + program $^{28}$ was mainly used to analyze the simulations of double strands.

The ptraj module of Amber was used to perform a clustering procedure of the n-ds trajectory data. The means algorithm was applied to produce 5 clusters using a RMS metric which compares all atoms in the central pentamer $\mathrm{A}_{7} \mathrm{~T}_{8} \mathrm{C}_{9} \mathrm{G}_{10} \mathrm{~T}_{11}$ duplex. The representative structure of the major cluster (population 33\%) was used in the quantum chemistry calculations.

The stacking between different bases was quantified by combining two parameters: the distance $\left(\mathbf{d}_{\mathbf{c e n}}\right)$ between the geometric centers of adjacent base rings and the angle $(\boldsymbol{\theta})$ between the vectors normal to the mean planes of the bases. The bases along the trajectory showing a geometry with $\mathbf{d}_{\text {cen }}<4.0 \AA$ and $\boldsymbol{\theta}<30^{\circ}$ (or $\boldsymbol{\theta}>150^{\circ}$ ) were considered to be stacked.

We also analyzed two distinctive distances between adjacent $\mathrm{T}$ and $\mathrm{C} / 5 \mathrm{mC}$ nucleobases along one strand: the distance (d)between midpoints of the C5-C6 bonds of adjacent $\mathrm{T}$ and $\mathrm{C} / 5 \mathrm{mC}$ rings and the distance $(\mathbf{g})$ between the $\mathrm{C} 5$ atom of $\mathrm{T}$ and the $\mathrm{N} 4$ atom of $\mathrm{C} / 5 \mathrm{mC}$. These two distances have been associated to CPD and 64PP formation, respectively. ${ }^{14}$

Quantum mechanical calculations. QM calculations were carried out by the Time Dependent (TD) DFT method, adopting M052X functional and including the solvent effect by the Polarizable Continuum Model (PCM).This approach has been already applied in the study of the excited states of oligonucleotides as well as their photoreactivity and, in particular on pyrimidine dimerization, providing results fully consistent with the experimental observations. ${ }^{9,}{ }^{29}$ Starting from a representative structure derived from MD simulations on $\mathrm{n}$-ds as described in the previous 
section, we extracted the central double stranded structure with three base pairs, $(\mathrm{TCG})_{\mathrm{ds} .}$ Subsequently, we performed a preliminary geometry optimization, in order to compensate possible inaccuracy in the determination of the base structureby MD simulations. To this end, we useda relatively high(0.003 a.u.) convergence threshold so that to keep the duplex conformation as close as possible to that issuing from MD simulations, which corresponds to the entire duplex and not only to a small fragment. A similar procedure was followed in the study of the single-stranded fragments $(\text { TCGTA })_{\mathrm{ss}}$ and $(\mathrm{ATCGT})_{\mathrm{ss}}$. The effect of methylation was checked simply by substituting the hydrogen atom bonded to C5 of cytosine by a methyl group.

\section{EXPERIMENTAL RESULTS}

\section{Dimerization quantum yields}

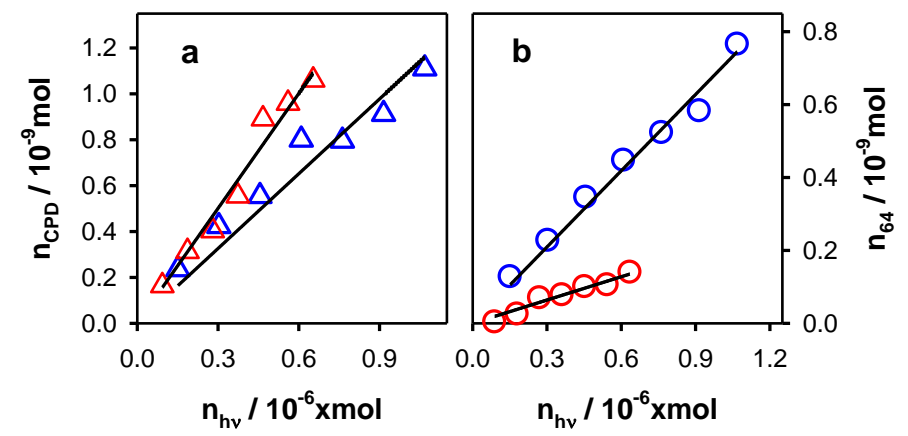

Figure 2. Effect of C5-methylation on the amount of $c, s$ CPDs (a) and 64PPS (b) detected as a function of the absorbed photons by n-ds (blue) andm-ds (red). Irradiation wavelength: $282 \mathrm{~nm}$.

We determined the quantum yields $\phi$ of dimeric photoproducts, CPDs and 64PPs, by adding both the $\mathrm{T} 5 \mathrm{mC}$ and $\mathrm{TC}$ derivatives and their deaminated TT counterparts. The concentration of detected photoproducts varied linearly with the number of absorbed photons, ruling out occurrence of secondary photoreactions such as photoreversion of CPDs or conversion of 64PPs into their Dewar valence isomers. For each system, at least two series of irradiations were carried out. An example is shown in Figure 2 while additional data are presented in Figures SI-2 and SI- 
3. The $\phi$ values were determined from the slopes of the linear regressions. As only $c, s$ CPDs were detected in duplexes, the values of $\phi_{\mathrm{CPD}}$ given below refer to only this diastereoisomer. However, we notethat $t, s$ CPDs are formed in single strands. Their ratio in respect to $c, s$ CPDs depends strongly on C5-methylation, dropping from 1/6 for n-ss and 1/16for m-ss.

Table 1.Quantum yields $\phi\left(\times 10^{3}\right)$ determined for the formation of dimeric photoproducts following irradiation of single and double strands at wavelength $\lambda_{\text {irr }}$.

\begin{tabular}{cccccc} 
& $\lambda_{\text {irr }}(\mathbf{n m})$ & $\mathbf{n}$-ss & $\mathbf{m}$-ss & $\mathbf{n}$-ds & m-ds \\
\hline$\phi_{\text {CPD }}{ }^{¥}$ & 255 & $1.32 \pm 0.03$ & $1.30 \pm 0.06$ & $0.62 \pm 0.03$ & $1.12 \pm 0.04$ \\
& 267 & $2.10 \pm 0.09$ & $2.14 \pm 0.05$ & $1.19 \pm 0.05$ & $1.31 \pm 0.04$ \\
& 282 & $1.49 \pm 0.05$ & $2.49 \pm 0.09$ & $1.09 \pm 0.05$ & $1.67 \pm 0.06$ \\
\hline \multirow{2}{*}{$\phi_{64}$} & 255 & $1.41 \pm 0.06$ & $0.29 \pm 0.02$ & $0.67 \pm 0.02$ & $0.15 \pm 0.02$ \\
& 267 & $2.93 \pm 0.11$ & $0.46 \pm 0.02$ & $0.72 \pm 0.01$ & $0.15 \pm 0.01$ \\
${ }^{*} c, s$ CPDs & 282 & $1.27 \pm 0.05$ & $0.46 \pm 0.03$ & $0.70 \pm 0.02$ & $0.21 \pm 0.01$
\end{tabular}

We observe in Table 1 that, depending on the system and the irradiation wavelength, C5methylation may have a negligible effect on $\phi_{\mathrm{CPD}}($ single strands, $255 \mathrm{~nm})$,or induce an increase up to $80 \%$ (duplexes, $255 \mathrm{~nm}$ ). The opposite effect is encountered for $\phi_{64}$, which decreases by a factor ranging from 3.3 to 6.6. We also remark that, regardless methylation, base-pairing reduces both $\phi_{\mathrm{CPD}}$ and $\phi_{64}$. Moreover, the variation of $\phi$ values is non-monotonous with the irradiation wavelength.

The values in Table 1 do not account directly for the specific reactivity of $5 \mathrm{mC}$ because all bases absorb at the irradiation wavelengths. ${ }^{16,30}$ Such a blurring is more pronounced for double than for single strands due to the presence of the non-reactive strand c-ss. In order to examine 
whether the differences between methylated and non-methylated systems arise simply from changes in the cytosine absorption spectrum upon C5-methylation, we make the hypothesis that the absorption spectrum of each monomeric chromophore within the single and double strands is the same as that of the monomeric chromophore in solution. Accordingly, we defined by the parameter $\mathrm{I}_{\mathrm{m}}=\phi_{\mathrm{m}} \psi_{\mathrm{m}} / \phi_{\mathrm{n}} \psi_{\mathrm{m}} ; \phi_{\mathrm{m}}$ and $\phi_{\mathrm{n}}$ are the quantum yields found for the a methylated and the corresponding non-methylated system, respectively; $\psi_{\mathrm{m}}$ and $\psi_{\mathrm{n}}$ represent the fraction of photons absorbed by a reactive $5 \mathrm{mC}$ or $\mathrm{C}$ of the TCG/T5mCG sequences (TableSI-1). Thus, the $\mathrm{I}_{\mathrm{m}}$ values, shown inTable 2, represent the effect of methylation on the quantum yields corrected for the absorption of reactive cytidines.

Table 2. Effect of C5-methylation on the quantum yields of dimeric photoproducts quantified by the parameter $I_{m}=\phi_{m} \psi_{n} / \phi_{n} \psi_{m}{ }^{y}$ accounting for the different absorption spectra of $\mathrm{C}$ and $5 \mathrm{mC}$

\begin{tabular}{cccc} 
& $\boldsymbol{\lambda}_{\text {irr }}(\mathbf{n m})$ & single strands & duplexes \\
\hline \multirow{3}{*}{ CPDs $^{*}$} & 255 & 1.3 & 2.4 \\
& 267 & 1.3 & 1.4 \\
& 282 & 1.4 & 1.3 \\
\hline \multirow{2}{*}{ 64PPs $^{\S}$} & 255 & 0.3 & 0.3 \\
& 267 & 0.2 & 0.3 \\
& 282 & 0.3 & 0.2
\end{tabular}

${ }^{*} \phi_{m}$ and $\phi_{n}$ are the quantum yields found for the methylated and the corresponding non-methylated system, respectively (Table 1); $\psi_{\mathrm{m}}$ and $\psi_{\mathrm{n}}$ represent the fraction of photons absorbed by a reactive $5 \mathrm{mC}$ or C of the TCG/T5mCG sequences (Table SI-1). Estimated errors: ${ }^{*} \pm 0.1,{ }^{\S} \pm 0.05$.

The $\mathrm{I}_{\mathrm{m}}$ values of CPDs are higher than 1. They are all around 1.3-1.4 with the exception the clearly higher value found for the $255 \mathrm{~nm}$ irradiation of duplexes. In contrast to CPDs, 64PPs are characterized by $\mathrm{I}_{\mathrm{m}}$ values lower than 1, around $0.2-0.3$.

At this stage, we draw the conclusion that the effect of C5-methylation on pyrimidine dimerization cannot be explained simply in terms of spectral changes of excited states localized 
on individual bases. Particularly intriguing is the enhancement of CPD formation for the short irradiation wavelength encountered only for duplexes.

\section{Fluorescence spectra}

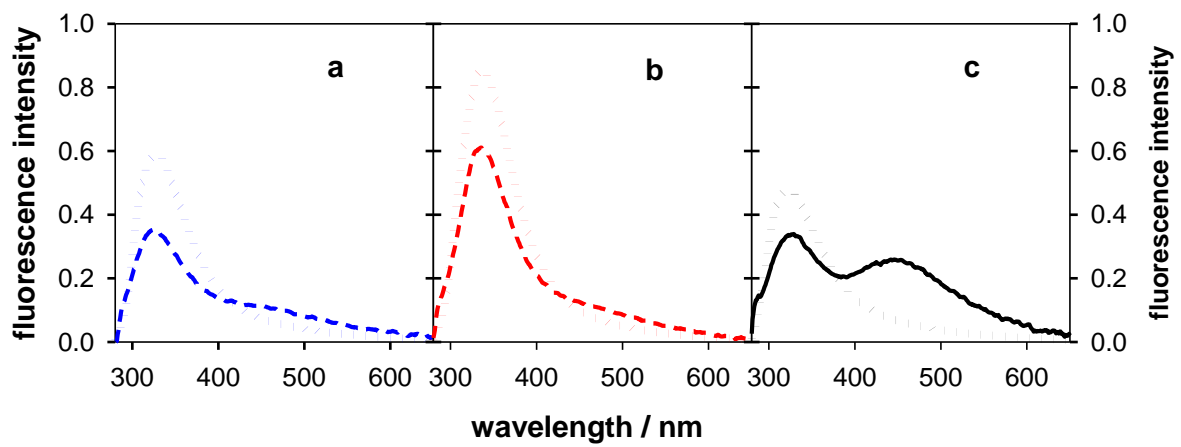

Figure 3. Comparison of the fluorescence spectra of the single stands (dashed lines), n-ss (a, blue), m-ss (b, red) and c-ss (c, black) with those of the corresponding stoichiometric mixture of nucleosides (dots). The spectral intensities are representative of the quantum yields. Excitation wavelength: $255 \mathrm{~nm}$.

The steady-state fluorescence spectra of the examined single and double strands exhibit a main emission band around $330 \mathrm{~nm}$ and a less intense feature, shoulder or well-defined band, around $425 \mathrm{~nm}$. The spectral changes observed upon varying the excitation wavelength from 255 to $282 \mathrm{~nm}$ are smaller than the experimental errors. The multimer spectra, together with those corresponding to stoichiometric mixtures of non-interacting monomers, are presented in Figures 3 and 4; their intensities are proportional to the fluorescence quantum yields $\phi_{\mathrm{f}}$, ranging from $0.6 \times 10^{-4}$ to $1.3 \times 10^{-4}$ (Table 3 ).

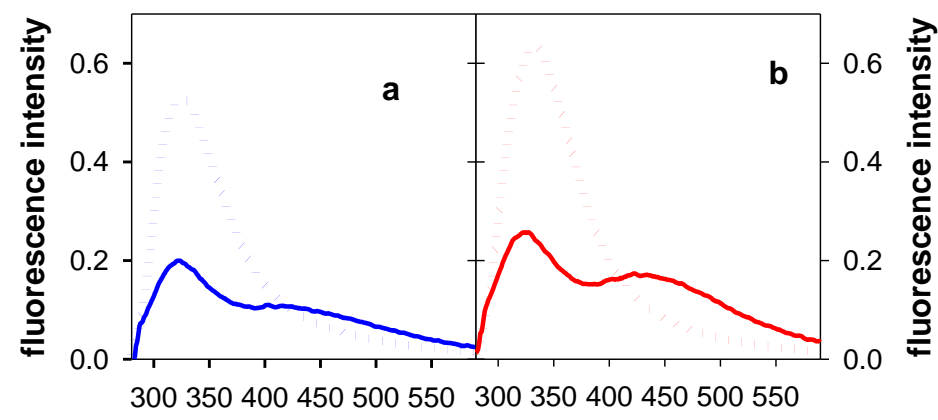

wavelength / nm 
Figure 4. Comparison of the fluorescence spectra of the duplexes (solid lines) $n$-ds and m-ds with those of the corresponding stoichiometric mixture of nucleosides (dots); (a, blue) non-methylated systems; (b, red) methylated systems. The spectral intensities are representative of the fluorescence quantum yields. Excitation wavelength: 255 $\mathrm{nm}$.

The position of the short wavelength band indicates that it corresponds to the $\pi \pi^{*}$ transition of monomers. Its intensity in the multimer spectra is lower than that observed for non-interacting monomers. We quantified this quenching using the parameter $\mathrm{Q}_{\pi \pi^{*}}=\left(\mathrm{I}_{\mathrm{s}}-\mathrm{I}_{\mathrm{M}}\right) / \mathrm{I}_{\mathrm{M}}$, where $\mathrm{I}_{\mathrm{s}}$ and $\mathrm{I}_{\mathrm{M}}$ denote the maximum intensity of the $\pi \pi^{*}$ emission band for the examined strand and the corresponding stoichiometric mixture of monomers (Figures 3 and 4). The absolute $\mathrm{Q}_{\pi \pi^{*}}$ values found for the duplexes amount to $\approx 0.6$ and are higher than those of single strands (Table 3 ). We also remark that the peak of m-ds spectrum is blue shifted $(324 \mathrm{~nm})$ compared to that of the corresponding monomers $(332 \mathrm{~nm})$, whereas for the other three systems this shift is within the experimental error bars.

The maximum difference between the multimer and monomer emission spectra appears at $455 \mathrm{~nm}$. Therefore, in analogy with $\mathrm{Q}_{\pi \pi^{*}}$, we defined the enhancement of the long wavelength fluorescence of the single and double strands by $\mathrm{E}_{455 \mathrm{~nm}}=\left(\mathrm{I}_{\mathrm{s}}-\mathrm{I}_{\mathrm{M}}\right) / \mathrm{I}_{\mathrm{M}}$, considering the intensities of the monomer and multimer spectra at $455 \mathrm{~nm}$. As shown in Table 3, the $\mathrm{E}_{455 \mathrm{~nm}}$ values range from 0.31 to 3.12 , being particularly high for the complementary single strand c-ss.

Our fluorescence study revealed an important redistribution of the excitation energy in the examined multimers, quenching of the $\pi \pi^{*}$ states and population of low lying charge transfer states, as attested by the appearance of emission bands in the visible region. These effects depend strongly on base-pairing (Figure SI-4). However, such observations do not allow discriminating fluorescence arising from the various types of bases. Yet, the blue shift exhibited by the maximum of the m-ds spectrum compared to that of monomers indicates strong quenching of the $5 \mathrm{mC}$ fluorescence, because this base emits at longer wavelengths than the others. ${ }^{30}$ 
Table 3. Fluorescence properties of the examined multimers observed for excitation at $255 \mathrm{~nm}$ : fluorescence quantum yields $\left(\phi_{\mathrm{f}}\right)$, fluorescence maximum $\left(\lambda_{\max }, \mathrm{nm}\right)$, quenching of the $\pi \pi^{*}$ fluorescence $Q_{\pi \pi^{*}}=\left(I_{s}-I_{M}\right) / I_{M}{ }^{¥}$ and enhancement $E_{455 n m}=\left(I_{s}-I_{M}\right) / I_{M}{ }^{\ddagger}$ of the emission at 455 nm.

\begin{tabular}{|c|c|c|c|c|c|}
\hline & n-ss & m-SS & c-ss & n-ds & m-ds \\
\hline$\phi_{\mathrm{f}}\left(\mathbf{x 1 0 ^ { - 4 }}\right)$ & 0.9 & 1.3 & 1.3 & 0.6 & 0.9 \\
\hline $\mathbf{Q}_{\pi \pi^{*}}^{\#}$ & -0.41 & -0.29 & -0.28 & -0.63 & -0.60 \\
\hline $\begin{array}{c}\lambda_{\text {max }, \mathbf{M}}{ }^{*} \\
\lambda_{\text {max }, \mathbf{s}}\end{array}$ & $\begin{array}{l}328 \\
324\end{array}$ & $\begin{array}{l}338 \\
336\end{array}$ & $\begin{array}{l}329 \\
328\end{array}$ & $\begin{array}{l}326 \\
323\end{array}$ & $\begin{array}{l}332 \\
324\end{array}$ \\
\hline $\mathbf{E}_{455 \mathrm{~nm}}^{\#}$ & +0.61 & +0.31 & +3.12 & +0.41 & +1.11 \\
\hline
\end{tabular}

${ }^{\mathrm{z}} \mathrm{I}$ indicates the intensity at the fluorescence maximum and at $455 \mathrm{~nm}$, for $\mathrm{Q}_{\pi \pi^{*}}$ and $\mathrm{E}_{455 \mathrm{~nm}}$, respectively (Figures 3 and 4 ). The indexes $\mathrm{s}$ and $\mathrm{M}$ refer to the examined multimer and the corresponding stoichiometric mixture of mono-nucleosides, respectively. Error bars: ${ }^{\#} \pm 0.10 ;{ }^{\ddagger} \pm 2 \mathrm{~nm}$.

\section{THEORETICAL RESULTS}

\section{Conformational factors}

Single-strands.The single-stranded structures fluctuate around B-DNA conformations, with the backbone angles that populate mainly canonical B-DNA values. For most of the trajectory time, the single strands maintain a base stacked helical structure. However, unstacking events frequently occur, also leading to the formation of folded hairpin structures (see SI and Figure SI5) with unusual dynamic H-bonds between bases along the curved strand. The formation of similar coiled states was already observed in independent MD simulations of other sequences in single strand conformation. ${ }^{31}$ The structural rearrangement and the variability of conformers sampled along the m-sstrajectory are illustrated in Figures 5a and 5b, where the starting structure and the structure closest to the average are shown (see also Figure SI-6).

The comparison of $\mathrm{n}$-ss and m-ss trajectories highlights important features that can be correlated to the photodimerization trends. The stacking of two consecutive bases in the strand was evaluated by calculating the distance $\mathbf{d}_{\mathrm{cen}}$ between the centers of their aromatic moieties and 
the angle between the vectors normal to the mean planes of the bases as described in the Computational Details section).We found that C5-methylation destabilizes CG stacking; the percentage of the CG stacked state is only $65 \%$ in m-ss vs $83 \%$ in n-ss (see SI and Table SI-2). In parallel, it increases the population of TC stacked states from $66 \%$ for n-ss to $70 \%$ for m-ss, thus altering the relative stability of $\mathrm{CG}$ and TC stacking.

$\mathbf{a}$

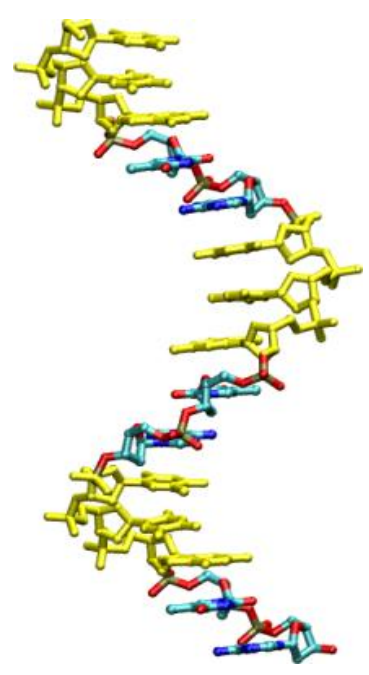

b

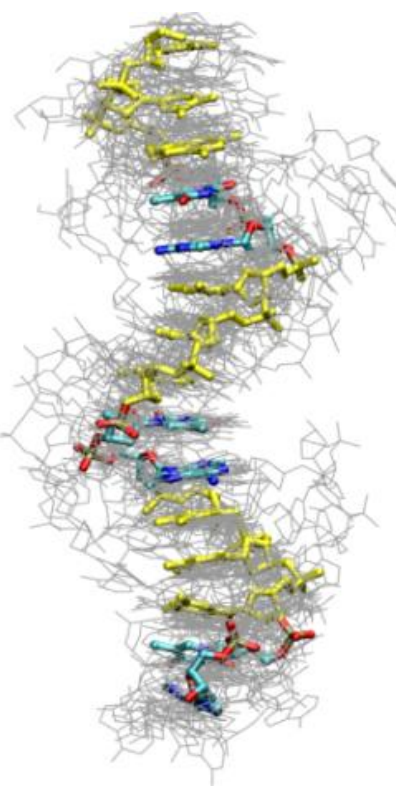

c

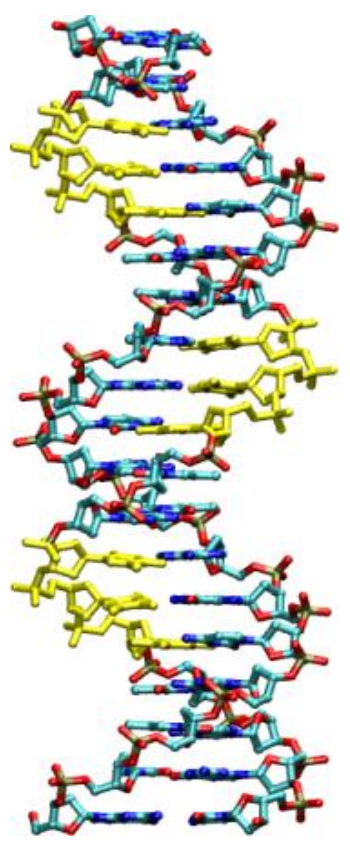

d

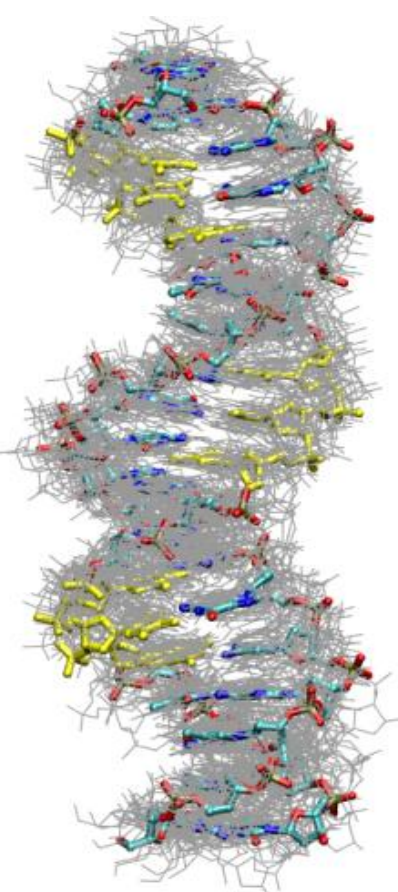

Figure 5. Structures derived frommolecular dynamicssimulations. Methylated single strand (a and b) and methylated duplex (c and d); (a) and (c):starting structures in whichthe hydrogen atoms are omitted for clarity; (b) and (d): structures closest to the average calculated on all non-hydrogen atoms (highlighted in ball and stick) superimposed on 20 structures (light gray) extracted from the trajectory every $25 \mathrm{~ns}$. The T5mCG triplets are depicted in yellow.

C5-methylation affects significantly the deoxyribose puckering of the deoxycytidine nucleotide, shifting the phase angle to smaller values, from 145.8 to 141.2 (average values for the three deoxycytidines present in the strand; Table SI-3). We further analyzed the distribution of the most populated conformers, i.e. C2'endo and C1'-exo, over the $500 \mathrm{~ns}$ trajectories sampled every 5ps (Table SI-4). In the case of n-ss, $54 \%$ of the deoxycytidines assume the 
C2'endo conformation whereas $42 \%$ of them assume the C1'exo conformation. The ratio is reversed in $\mathrm{m}$-ss, where the $\mathrm{C} 2$ 'endo and $\mathrm{C} 1$ 'exo populations are, on average, $43 \%$ and $53 \%$, respectively.

In the MD ensembles we have also analyzed the distance between midpoints of the C5-C6 bonds (d) of adjacent $\mathrm{T}$ and $\mathrm{C}$ rings, which has been related with $\mathrm{CPD}$ formation. ${ }^{14}$ The distances are, on average, shorter for the T5mC pairs (4.20 ̊) than for the TC pairs (4.44 $\AA$ ) (Table 4). In addition, the fraction of short distances $(<4.0 \AA)$ is higher in m-ss $(45 \%)$ than in n-ss $(37 \%)$ (Table SI-5). We carried out a similar analysis on the distance between the C5 atom of T and the $\mathrm{N} 4$ atom of $\mathrm{C} / 5 \mathrm{mC}(\mathbf{g})$, which has been related with the formation of $64 \mathrm{PPs} .{ }^{14}$ In this case, the fraction of short distances $(<4.0 \AA)$ is only marginally higher in n-ss $(38 \%)$ than in the m-ss (37\%) (Table SI-6).

Duplexes. Both methylated and non-methylated duplexes reached a stable state in 500ns of simulation (see SI for details). The overall average RMSDs on non-hydrogen atoms from the starting structure are $3.2 \pm 0.6 \AA$ and $3.1 \pm 0.6 \AA$ for $\mathrm{n}$-ds and m-ds, respectively. The TCG sequences along the $5^{\prime} \rightarrow 3^{\prime}$ strand adopt a typical B-DNA state. In Figure $5 \mathrm{c}$ and $5 \mathrm{~d}$ are reported the starting structure and the structure closest to the average structure calculated along the trajectory. We note that the duplexes adopt a highly stable structure without significant unstacking events along the trajectory. C5-methylation increases the stiffness of the duplex, as shown by the analysis of the RMSF (Root Mean Square Fluctuations) in Figure SI-7. . The increased rigidity of DNA upon C5-methylation was also predicted by previous theoretical works. ${ }^{32-33}$ and has been recently confirmed by a combined experimental/MD study. ${ }^{34}$ Further minor differences induced by methylation (i.e. BI/BII distribution and base twist; see SI and Figure SI-8) are in agreement with previously reported MD and experimental data. ${ }^{32,35}$ 
Table 4. Average distances (d) in (̊̊) between the midpoints of C5-C6 bondsin TC/T5mC pairs. Values are calculated over the 500000 structures in each trajectory. The standard deviations are shown in brackets.

\begin{tabular}{|c|c|c|c|}
\hline \multicolumn{2}{|c|}{ n-sS } & \multicolumn{2}{|c|}{ m-SS } \\
\hline T1-C2 & $4.53(1.50)$ & $\mathrm{T} 1-5 \mathrm{mC} 2$ & $4.16(0.60)$ \\
\hline T6-C7 & $4.61(1.95)$ & T6-5mC7 & 4.38 (1.08) \\
\hline T11-C12 & $4.17(0.53)$ & $\mathrm{T} 11-5 \mathrm{mC} 12$ & $4.06(0.43)$ \\
\hline all TC pairs ${ }^{¥}$ & $4.44(1.50)$ & $\begin{array}{c}\text { all } \mathrm{T} 5 \mathrm{mC} \\
\text { pairs }^{¥}\end{array}$ & $4.20(0.76)$ \\
\hline \multicolumn{2}{|c|}{ n-ds } & \multicolumn{2}{|c|}{ m-ds } \\
\hline T3-C4 & $4.19(0.39)$ & $\mathrm{T} 3-5 \mathrm{mC} 4$ & $4.25(0.32)$ \\
\hline T8-C9 & $4.26(0.41)$ & $\mathrm{T} 8-5 \mathrm{mC} 9$ & $4.28(0.33)$ \\
\hline T13-C14 & $4.26(0.41)$ & $\mathrm{T} 13-5 \mathrm{mC} 14$ & $4.28(0.33)$ \\
\hline all TC pairs ${ }^{¥}$ & $4.24(0.40)$ & all TC pairs ${ }^{¥}$ & $4.27(0.33)$ \\
\hline
\end{tabular}

We analyzed the stacking of consecutive bases in the reactive strand of duplexesusing the same geometric parameter as in single strands: the distanced $\mathbf{d}_{\text {cen }}$, between centers of the nucleobase rings.Due to the rigidity of the duplex, the filter of the angle between the vectors normal to the mean planes of the bases is not necessary. We found the C5-methylation does not affect significantly the stacking distance of nucleobases along a single strand. The $\mathbf{d}_{\text {cen }}$ corresponding to TC and CG pairs, averaged over the three pairs in the sequence, is essentially the same in methylated and non-methylated duplexes: $4.0 \AA$ forTC/T5mC; $4.35 \AA$ for $\mathrm{CG} / 5 \mathrm{mCG}$ (see SI for details). The analysis of sugar puckering reveals that C5-methylation decreases sugar phase angles of cytidine $\left(128.8^{\circ}\right.$ for $\mathrm{C}$ vs. $120.9^{\circ}$ for $\left.5 \mathrm{mC}\right)$ and favors population of $\mathrm{C} 1^{\prime}$ exo/O4'endo pucker over the C2'endo one (Table SI-7). A structural analysis of the TC and T5mC steps 
reveals that $\mathrm{C} 5$-methylation increases the population of $\mathrm{C} 1$ 'exo/ $\mathrm{O} 4$ '-endo and $\mathrm{C} 1$ 'exo/C1'exo conformers, allowing a closer approach of the reactive CC bonds (Table SI-8 and Table SI-9).

The average distances $\mathbf{d}$ do not show significant differences between methylated and nonmethylated structures (Table 4 and SI). However, the standard deviations of the d distributions are lower for $\mathrm{T} 5 \mathrm{mC}(0.33)$ than for TC $(0.40)$. This confirms that $\mathrm{C} 5$-methylation, by narrowing the distance distribution, increases the stiffness of the TC pairs. The $\mathbf{g}$ distances have also very similar average values in $\mathrm{n}-\mathrm{ds}$ and $\mathrm{m}-\mathrm{ds}$ simulations (Table SI-10 and Figure SI-9), but the fraction of duplexes adopting $\mathbf{g}$ distances shorter than $4.0 \AA$ is higher for $\mathrm{n}$-ds $(0.66)$ than for $\mathrm{m}$ ds (0.59).

The structures along the n-ds trajectory have been subjected to a clustering procedure. In particular, we used a RMS metric comparing all atoms in the central pentamer $\mathrm{A}_{7} \mathrm{TCGT}_{11}$ duplex. The representative structure of the major cluster, representing $33 \%$ of the population, was introduced in the QM calculations.

The main conclusion of our MD simulations is that C5-methylation favors TC stacking over CG stacking in single strands but this effect is weaker in duplexes. Nevertheless, the presence of three extra methyls in duplexes increases their stiffness and leads to a shift of the sugar puckering.

\section{Electronic factors}

In a first step, we examined the Franck-Condon excited states of the model duplexes $(\mathrm{TCG})_{\mathrm{ds}}$ and $(\mathrm{T} 5 \mathrm{mCG})_{\mathrm{ds}}$. The electronic density shifts associated to each of the 5 lowest electronic transitions of (TCG) $)_{\mathrm{ds}}$ are shown in Figure 6. Notwithstanding the limitations of our approach,which does not take into account geometry fluctuations and does not include all the bases present in the experimentally studied systems, we can draw some qualitative conclusions. 
Although the 20 lowest energy excited states are built with contribution from bases on both strands, the most important part of the excitation is always located on a single strand. The lowest energy excited $\pi \pi *$ transition of $\mathrm{C}$ is significantly mixed with the electronic transitions of the adjacent bases within the same strand. This is the case of the $S_{2}, S_{3}, S_{5}$ states, for which the electronic density is shown in Figure 6. We have also depicted in Figure 6 the weak transitions the $\mathrm{S}_{0} \rightarrow \mathrm{S}_{4}$ and $\mathrm{S}_{0} \rightarrow \mathrm{S}_{5}$, implicating the complementary single strand, with significant $\mathrm{G} \rightarrow \mathrm{C}$ and $\mathrm{G} \rightarrow \mathrm{A}$ charge transfer character.
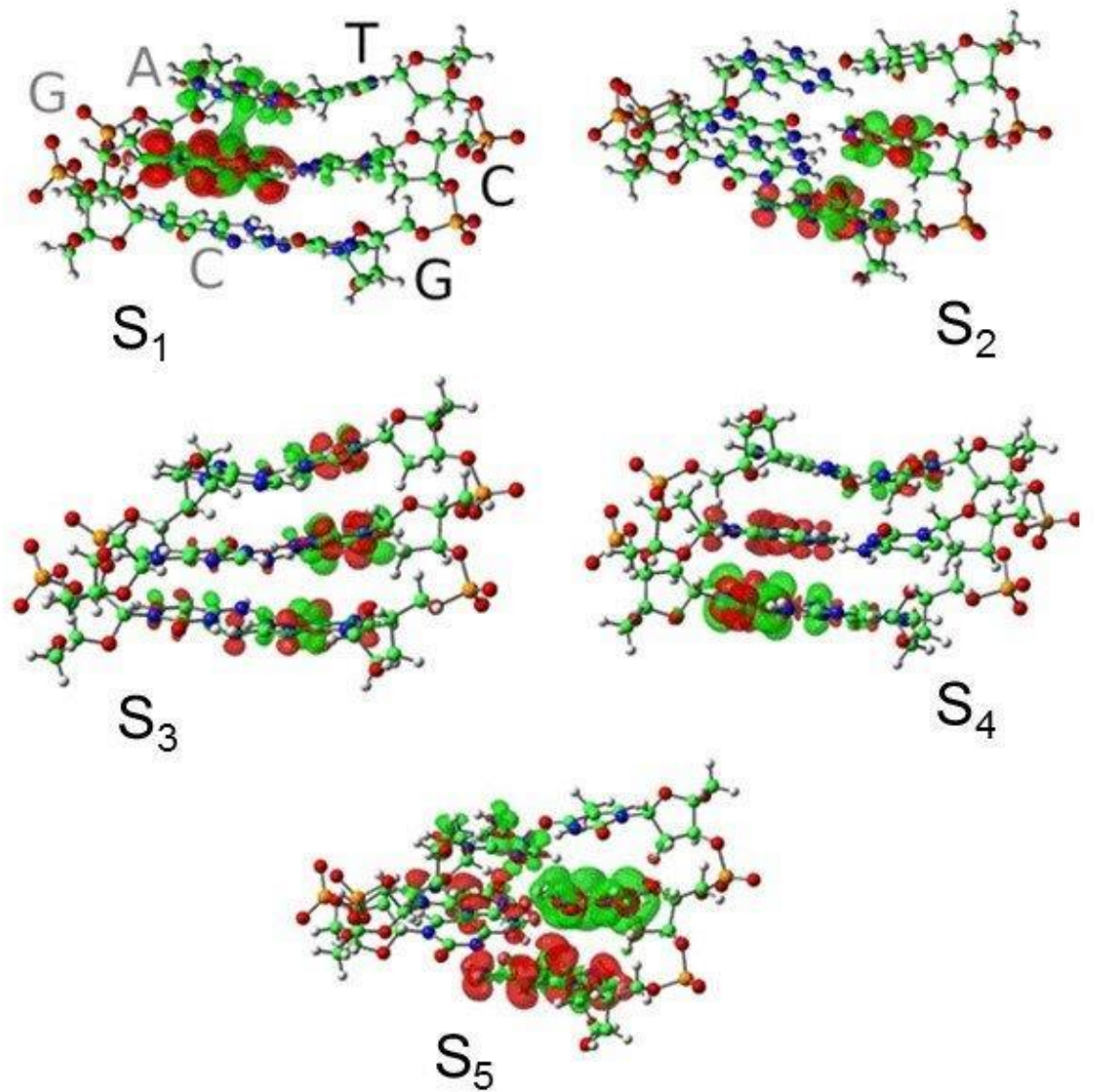

Figure 6. Schematic drawing of the electronic density difference associated to the five lowest electronic transitions in $(\mathrm{TCG})_{\mathrm{ds}}$ according to PCM/TD-M052X/6-31G(d) calculations. Red: decrease of the electron density; green: increase of the electron density.

The lowest energy transition in (T5mCG) $)_{\mathrm{ds}}$ can be described essentially as a $\pi \pi^{*}$ transition localized on $5 \mathrm{mC}$, which receives small contribution from other excitations (Figure 7a). The 
second excited state (Figure 7b) derives from the interaction between the $\mathrm{L}_{\mathrm{a}}$ excited state localized on the $\mathrm{G}$ on the complementary strand and the $\mathrm{G} \rightarrow \mathrm{A}$ charge transfer state. Similarly, $\mathrm{S}_{3}$ (Figure7c) is localized on the $\mathrm{G}$ of the methylated strand, but has a non-negligible $\mathrm{G} \rightarrow 5 \mathrm{mC}$ charge transfer character.
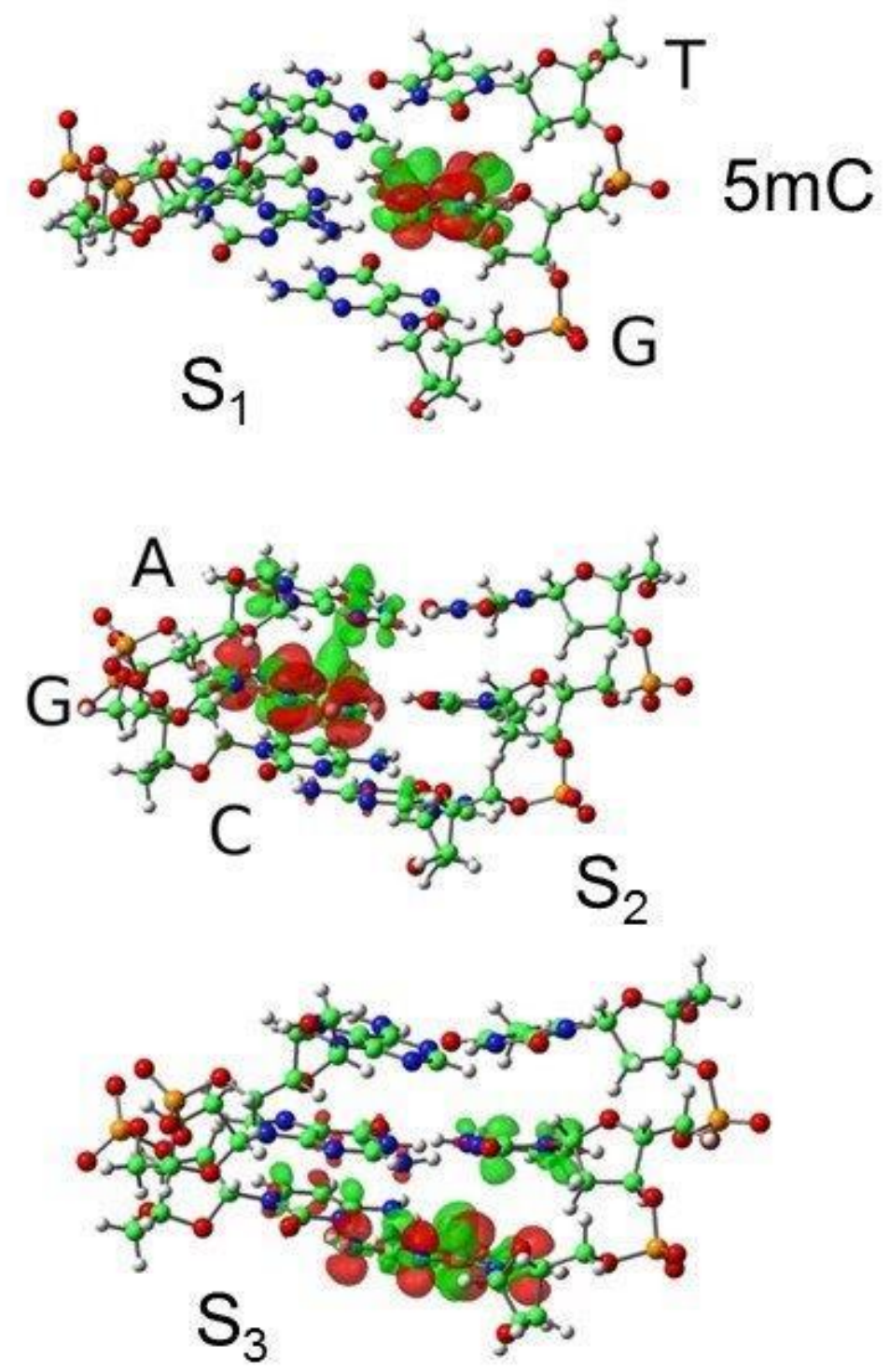

Figure 7. Schematic drawing of the electronic density difference associated to the three lowest electronic transitions in $(\mathrm{T} 5 \mathrm{mCG})_{\mathrm{ds}}$ according to PCM/TD-M052X/6-31G(d) calculations. Red: decrease of the electron density; green: increase of the electron density.

Since our calculations show that the electronic transitions mainly involve the bases of a single strand, we analyzed the Franck-Condon region of our two model single strands (TCGTA) $)_{\text {ss }}$ and 
$(\text { T5mCGTA })_{s s}$, always starting from structures derived from the duplex MD simulations. This analysis is expected to provide insights on the excited state behavior of both the single strand and duplex systems under examination. However, we stress that, in respect to single strands the weight of long stacked sequences are overestimated, while in respect to duplexes the excited states of the complementary strand are missing.
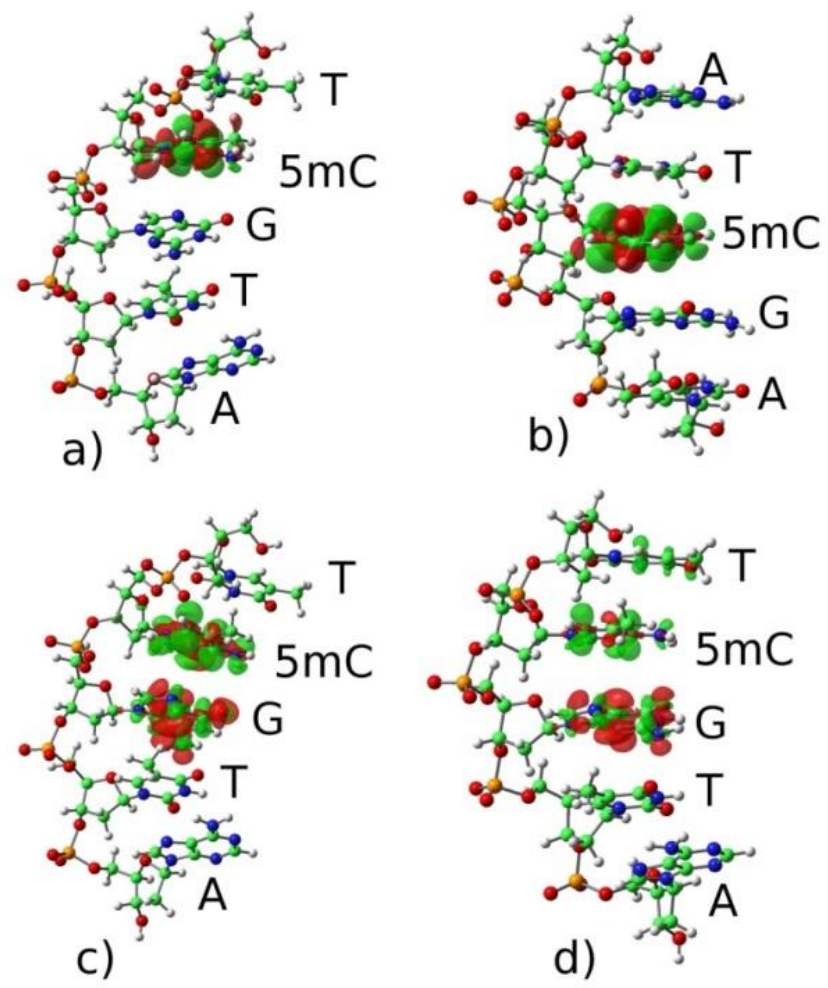

Figure 8. Schematic drawing of the electronic density difference associated to electronic transitions of model single strands in the n-ds geometry according to PCM/TD-M052X/6-31G(d) calculations. a) $S_{0} \rightarrow S_{1}$ in (T5mCGTA) ss; $\mathrm{S}_{0} \rightarrow \mathrm{S}_{1}$ in (AT5mCGT) ss $_{\text {s }}$ c) $\mathrm{S}_{0} \rightarrow \mathrm{S}_{13}$ in(T5mCGTA) $)_{\mathrm{ss}}$; d) $\mathrm{S}_{0} \rightarrow \mathrm{S}_{14}$ in (T5mCGTA) $)_{\mathrm{ss}}$. Red: decrease of the electron density; green: increase of the electron density.

The results on the non-methylated systems are reported in Figure SI-10. Figure8shows the electronic density shifts associated to some low-lying electronic transitions in two singlestranded methylated structures. For both examined sequences, the lowest energy transition is essentially (>99\%) localized on $5 \mathrm{mC}$. Interestingly, for (TCGTA) $)_{s s}$, although the $\mathrm{C}$ has the largest contribution to the lowest energy transition, it also contains a small participation of 
theT $\pi \pi^{*}$ excited state (Figure SI-10). Inboth the methylated and non-methylated single strands, G bases can also be coupled with an adjacent $\mathrm{T}$ base, as shown by the presence of a low-lying transition with significant $\mathrm{G}^{+} \rightarrow \mathrm{T}^{-}$charge transfer character. This transition is not present in the duplexes, containing three base pairs. The analysis of the higher lying transitions, that can be associated to the maximum of the experimental absorption peak, i.e. around $260 \mathrm{~nm}$, shows a significant coupling between the electronic transitions localized on different bases: G (essentially corresponding to the $\mathrm{L}_{\mathrm{b}}$ excited state), $\mathrm{T}$ and of $5 \mathrm{mC}$ (Figure $8 \mathrm{c}$ and $\mathrm{d}$ ).

\section{DISCUSSION}

The degree of C5-methylation of our model strands (20\% for single strands, $10 \%$ for duplexes) is higher than that encountered in genomic DNA, which may reach $3 \%$ in the tumor suppressor gene p53. ${ }^{8}$ Therefore, its effect on the determined quantum yields for pyrimidine dimerization should be larger compared to that expected for natural systems. Despite this fact, the increase in $\phi_{\mathrm{CPD}}$ is at most $80 \%$ (Table 1). Surprisingly, the highest increase (about a factor two) was found for the shortest irradiation wavelength. For comparison, we note that the maximum increase in the CPD yields reported for genomic DNA is $70 \%$ in the UVB region and practically no effect at $254 \mathrm{~nm} .{ }^{8}$ The difference with our results is probably due to the lower degree of C5-methylation of natural DNA and to the fact that the precise number of absorbed photons, measured in the determination of quantum yields is not taken into account in the determination of yields. As far as 64PPs are concerned, our results, showing that the C5methylation reduces drastically their quantum yield (Table 1), contrast with the only related study on duplexes which reported significant enhancement for both UVB and UVC irradiation. ${ }^{6}$

Our "corrected" quantum yields expressed by the $\mathrm{I}_{\mathrm{m}}$ values in Table 2 clearly show that the effect of C5-methylation on the UV-induced pyrimidine dimerization cannot be explained simply 
by the difference between the absorption spectra of $\mathrm{C}$ and $5 \mathrm{mC}$. If that was the case, all the $\mathrm{I}_{\mathrm{m}}$ values would be equal to one but this is not observed for either types of dimeric photoproducts, CPDs and 64PPs. Before discussing our results obtained by fluorescence spectroscopy, molecular dynamics simulations and quantum dynamical calculations, we recall the connection of the UV-induced reactivity with electronic excitations in DNA, as emerged from recent experimental and theoretical studies.

According to previous work, $\mathrm{CPD}$ formation is induced by absorption of UV radiation directly by DNA occurs via $\pi \pi^{*}$ states $^{9,29,36}$ while the reactions leading to oxetane and azetidine, which are precursors of TT and TC 64PPs, respectively, take place in a charge transfer state. ${ }^{9,} 29$ Therefore, the population of the reactive excited states is expected to play a key role in this reactivity. It may be added thatelectronic excitations within DNA multimers are redistributed during their lifetime among different types of excited states. ${ }^{37}$ On the one hand, an ultrafast energy transfer, involving Franck-Condon excited states delocalized over several bases (excitons), takes place among various $\pi \pi^{*}$ states of the bases in model duplexes and natural DNA. ${ }^{13,} 38$ On the other hand, $\pi \pi^{*}$ states may evolve toward charge transfer states. ${ }^{39-41}$ In addition to these electronic factors, the appropriate geometrical arrangement ${ }^{14}$ of the reactive bases, determined among others by the sugar puckering, is crucial and so is the capacity of the strand to structurally accommodate the final photoproduct. In addition to these "direct" structural effects, the helix conformation plays also an "indirect" role by affecting the fate of collective excited states.

With the above general considerations in mind, we first focus on CPDs starting from single strands. The effect of C5-methylation on CPD formation in these relatively flexible structures is uniform for all the irradiation wavelength $\left(\mathrm{I}_{\mathrm{m}} \sim 1.3\right.$; Table 2$)$, suggesting that the reaction is 
controlled by "direct" conformational effects. Their characterization is thus important for understanding the behavior of duplexes in which more complex processes intervene, as indicated by the wavelength dependence of $\mathrm{I}_{\mathrm{m}}$.

One important origin of the observed increase in $\phi_{\mathrm{CPD}}$ in single strands is related to the sugar puckering. ${ }^{9,}{ }^{42}$ As a matter of fact, the presence of an extra methyl in cytosine favors geometrical arrangements of $\mathrm{T}$ and $\mathrm{C}$ that are likely to react. This trend was theoretically demonstrated for TCG/T5mCG tri-nucleotides and was found to be independent of the presence of guanine, ${ }^{9}$ in line with the increase of the CPD yield observed also in TC di-nucleotides. ${ }^{43}$ In this respect it is interesting to compare in a quantitative way the results obtained previously for tri-nucleotides and those found for single strands. Our molecular dynamics simulations have shown that C5methylation modifies the deoxyribose puckering of the cytidine nucleoside by shifting the phase angle to smaller values. The difference between the methylated and non-methylated cytosine puckering is $9^{\circ}$ in tri-nucleotides whereas in single strands it is only $4.6^{\circ}$ because the presence of the other bases in the larger system affect the local geometry of the TC step. This "direct" structural effect is reflected in the $\mathrm{I}_{\mathrm{m}}$ values determined for the tri-nucleotides (1.7; Table 1 in ref. 9) and single strands (1.3; Table 2), respectively. The better TC stacking in m-ss explains also the lower quantum yield found for $t, s$ CPDs compared to n-ss because their formation requires unstacking events of the TC pair leading from parallel to anti-parallel alignment of the reacting pyrimidines.

Another effect to be considered in CPD formation is that of the flanking base. It was reported that the quantum yield of TT CPDs in double-stranded structures is affected by the type of bases flanking the reactive di-nucleotides. ${ }^{44}$ This happens because the dimerization reaction is in competition with the formation of charge transfer states between one reactive thymine and the 
adjacent flanking base, the oxidation potential of the latter being a decisive parameter to the issue. ${ }^{15}$ In the case of TCG tri-nucleotides, formation of TC CPDs is in competition with the evolution of $\pi \pi^{*}$ states toward $\mathrm{C}^{-} \rightarrow \mathrm{G}^{+}$charge transfer states. ${ }^{9}$ The presence of an extra methyl at the 5 position of cytosine, destabilizes the CG stacking, thus favoring instead CPD formation. ${ }^{9}$ According to our MD simulations C5-methylation decreases the CG stacked conformations by $22 \%$ (versus $30 \%$ for tri-nucleotides). The fingerprint of this process is reflected in the quenching of the $\pi \pi^{*}$ fluorescence in $\mathrm{m}$-ss, which is $29 \%$ lower compared to that observed for $\mathrm{n}$ ss (Table 3). Although fluorescence arises potentially from all types of bases, the fact that the $\mathrm{m}$ ss fluorescence spectrum peaks at $338 \mathrm{~nm}$ (Table 3) indicates contribution of the $5 \mathrm{mC}$, whose fluorescence spectrum is red-shifted $\left(\lambda_{\max }=342 \mathrm{~nm} \text { for the corresponding nucleoside }\right)^{30}$ compared to those of other nucleosides $\left(\lambda_{\max }=307,330,328\right.$ and $334 \mathrm{~nm}$ for $\mathrm{dA}, \mathrm{dT}, \mathrm{dC}$ and $\mathrm{dG}$, respectively). ${ }^{16}$ In addition to these structural factors, the presence of an extra methyl on cytosine reduces its electron affinity and, consequently, the propensity to participate to a $\mathrm{C}^{-} \rightarrow \mathrm{G}^{+}$ charge transfer state.

In duplexes, the "direct" structural parameters that are important for CPD formation are less impacted by the presence of an extra methyl on the reactive cytosines with respect to single strands. Moreover, there are no important differences in the stacking distance for all the bases between $\mathrm{n}$-ds and $\mathrm{m}$-ds, in line with the similar quenching of $\pi \pi^{*}$ fluorescence in the two systems (Table 3). Despite this similarity, C5-methylation affects the sugar puckering associated with $5 \mathrm{mC}$, leading to an average decrease of the phase angle (Table SI-7) and increasing the population of bipyrimidine conformations characterized by closer reactive bonds(Tables SI- 8 and SI-9). Moreover, the lower electron affinity of $5 \mathrm{mC}$ is expected to disfavor evolution of $\pi \pi^{*}$ states toward $\mathrm{C}^{-} \rightarrow \mathrm{G}^{+}$charge transfer states, as in the case of single strands. 
Along with the processes related to population of charge transfer excited states, further effects related to exciton states may influence CPD formation in duplexes. Our quantum chemistry calculations on fragments of the examined duplexes have shown indeed that Franck-Condon excited states may extend over two or more bases. According to studies performed in the frame of the exciton theory for duplexes with different base sequences, delocalization persists even in the presence of conformational disorder. ${ }^{45}$ In a perfectly rigid system, internal conversion among exciton states (intraband scattering) leads to the bottom of the exciton band. Intraband scattering corresponds to energy transfer among the bases because the topography of upper and lower exciton states is not the same. ${ }^{46-47}$ In other terms, different bases contribute to upper and lower states (Figures 7 and 8 ). In the presence of conformational motions, there is competition between intraband scattering and localization of the $\pi \pi^{*}$ excitations on single bases, possibly with further evolution toward charge transfer states. Two limiting scenarios illustrating relaxation of exciton states for rigid and flexible systems are depicted schematically in Figure 9.

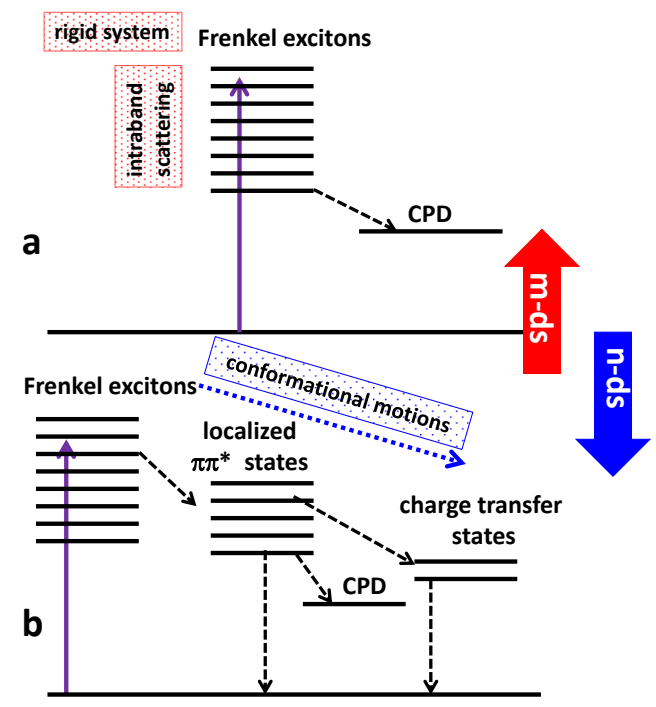

Figure 9. Effect of the duplex rigidity on the intraband scattering and its consequence to CPD formation. In rigid systems (a), intraband scattering leads to the bottom of the exciton band, transferring the excitation energy to reactive bases. In the presence of conformational motions (b), intraband scattering is in competition with localization 
of $\pi \pi^{*}$ excitations, occurring randomly on various bases, including non-reactive ones, and possible evolution to charge transfer states. C5-methylation increases the duplex rigidity favoring transfer of $\pi \pi *$ excitations from initially populated collective states to reactive pyrimidines.

As a result ofintraband scattering in m-ds reactive pyrimidines will receive the energy absorbed simultaneously by several bases. The efficiency of energy transfer depends on the number of bases participating to the Franck-Condon excited state populated by photon absorption. The participation ratio (or in other terms the degree of delocalization) is smaller for the exciton states located in the red part of the absorption spectrum, ${ }^{48}$ explaining the decrease of $\mathrm{I}_{\mathrm{m}}$ with increasing irradiation wavelength. According to our molecular dynamics simulations the structure of n-ds is less rigid, in the sense that the dynamical fluctuations are larger (Figure SI-7) than that of m-ds, in line with the higher melting temperature of the methylated duplex (Figure SI-1). For n-ds, which is less rigid than the m-ds, intraband scattering will be less efficient. As the competing process, localization of the excitation, occurs randomly in any of the bases participating to the initially populated collective state, the probability of energy transfer to reactive pyrimidines is lower in $\mathrm{n}$-ds compared to $\mathrm{m}$-ds.

It is also interesting to compare our results on CPDs with those obtained using locked configurations of thymines. ${ }^{49}$ It was found that such an increase in the stiffness of reactive pyrimidines induces a larger increase in the $\phi_{\mathrm{CPD}}$ in single than in double stranded structures. This effect was correlated with the distances in the reactive bonds in such system, determined by molecular dynamics simulations. The fact that the opposite effect is observed for methylated systems examined here, in particular for $255 \mathrm{~nm}$ irradiation, corroborates the occurrence of an energy transfer and trapping process.

Regarding the effect of C5-methylation on 64PPs, as they result from a two-step reaction, involving an azetidine intermediate (Scheme 1), the quantum yields reflect the overall effect. The 
$I_{m}$ values corresponding to 64PPs are practically constant $\left(I_{m}=0.2-03\right)$ for all the irradiation wavelengths and they are not affected by base pairing (Table 2). Such a uniform behavior suggests that the reaction is not impacted by the important conformational differences between single and double strands. Instead, the factors controlling $\phi_{64}$ appear to be rather local, tightly connected with the reacting chromophores.

Local factors, intervening in the first step of the reaction, may be conformational. The conical intersection crucial for the formation of the azetidine intermediate exhibits a c2c4 puckering, which is destabilized in the presence of methyl. Additional indications are provided by the MD analysis of the distances $\mathbf{g}$, related to azetidine formation, which are slightly shifted to lower values for n-ds compared to m-ds (Table SI-10 and Figure SI-9). From an electronic point of view, the strong decrease of the cytosine ionization potential $(0.32 \mathrm{eV}$, according to $\mathrm{M} 052 \mathrm{X} / 6$ $31+G(d, p)$ calculations $)$ induced by the presence of the methyl suggests an increased stability of the non-reactive $\mathrm{C}^{+} \rightarrow \mathrm{T}^{-}$charge transfer state, and, therefore, a smaller propensity to give rise to $\mathrm{T}^{+} \rightarrow \mathrm{C}^{-}$charge transfer state, leading to azetidine. The formation of the latter intermediate implies $(2+2)$ cycloaddition between the C5-C6 bond of the thymine and the imine group of the cytosine whose electronegativity is may affect the reaction. Thus, an decrease in electronegativity, achieved by N4-methylation of C induces a tenfold increase in the yield of 64PPs in TCG trinucleotides. ${ }^{50}$ The presence of the methyl at the 5 position of cytosine is expected to reduce this electronegativity, rendering the cycloaddition more difficult. The role of the electronegativity in the stability of the reaction intermediate leading to 64PPs is supported by the results obtained following substitution of the Oxygen atom in the $\mathrm{C} 4 \mathrm{O} 8$ carbonyl group by the less electroengative Sulphur atom. Only thietane, the intermediate arising from cycloaddition between thymidine and 4-thiothymidine, was stable enough to be isolated and characterized. ${ }^{51}$ 
The final step leading to 64PPs in general, has been less studied. It was shown that, in the cases of thymine single strands, it takes place on the millisecond time scale. ${ }^{52}$ Theoretical calculations indicate that the oxetane ring opening requires participation of two water molecules from the local environment. We could infer that the presence of a methyl on cytosine modifies the water network in the vicinity of azetidine which participate to this reaction, but for the moment we have not clear proofs on this issue.

\section{CONCLUSION}

In the present study, we have explored various physicochemical factors underlying pyrimidine dimerization in C5-methylated duplexes containing the biologically relevant sequence TCG, associated with carcinogenic mutations. For the first time, the effect of C5-methylation on pyrimidine dimer formation in duplexes was quantified by determination of quantum yields, providing a solid ground for studies aimed at the understanding of the related fundamental process. We have found that the CPD quantum yield in methylated systems, in contrast to nonmethylated ones, increases with increasing irradiation wavelength, confirming that the red-shift of the cytosine absorption spectrum upon C5-methylation does play a role in this photo-reaction. However, this red shift accounts only partly for the examined reactivity because the effect of C5methylation on CPD formationin duplexes is clearly larger for shortest irradiation wavelength $(255 \mathrm{~nm})$, where the absorption of $5 \mathrm{mC}$ is less intense than that of C. Conformational changes, especially related to the sugar puckering, appear in fact to be important, stabilizing stacking arrangements that favor CPD formation while perturbing those leading to 64PPs. Structural changes are strongly connected to the redistribution of the excitation energy in double-stranded structures. On the one hand, they may affect the participation of $\mathrm{C}$ to excited charge transfer states with the neighboring bases $\left(\mathrm{T}^{+} \rightarrow \mathrm{C}^{-}, \mathrm{C}^{-} \rightarrow \mathrm{G}^{+}\right)$. On the other, they increase the duplex 
rigidity, a condition that is crucial for energy transfer from non-reactive bases to reactive ones, via delocalized excited states (excitons). Such a transfer could explain the better reactivity toward CPDs observed for $255 \mathrm{~nm}$ irradiation. 


\section{AUTHOR INFORMATION}

\section{Corresponding Authors}

thierry.douki@cea.fr

robimp@unina.it

dimitra.markovitsi@cea.fr

The authors declare no competing financial interests.

\section{ACKNOWLEDGMENT}

Financial support from the French Agency for Research (ANR-10-BLAN-0809-01 and ANR-12BS08-0001-01) andthe CNRS-CNR PICS project (N6827-2015).

\section{SUPPORTING INFORMATION AVAILABLE}

Experimental: melting curves;HPLC-MS/MS chromatograms; dimeric photoproducts versus absorbed photons; effect of C5-methylation on the absorption spectra of oligomers; fraction of photons absorbed by reaction $\mathrm{C}$ or $5 \mathrm{mC}$. Molecular dynamics simulations on single-strands: gyration radius; TC and CG base twist angles; base stacking analysis; sugar puckering; $\mathrm{d}$ and $\mathrm{g}$ distances. Molecular dynamics simulations on duplexes:Root Mean Square Fluctuations; $\mathrm{CG} / 5 \mathrm{mCG}$ twist; sugar puckering; $\mathrm{d}$ and $\mathrm{g}$ distances. Quantum mechanical calculations: electronic transitions of (TCGTA) $)_{\text {ss }}$. This information is available free of charge via the Internet at http://pubs.acs.org 


\section{REFERENCES}

(1) Klose, R.; Bird, A.; Genomic DNA Methylation: The Mark and Its Mediators. Trends Biochem Sci. 2006,31, 89-97

(2) Mikkelsen, T. S.; Hanna, J.; Zhang, X. L.; Ku, M. C.; Wernig, M.; Schorderet, P.; Bernstein, B. E.; Jaenisch, R.; Lander, E. S.; Meissner, A.; Dissecting Direct Reprogramming through Integrative Genomic Analysis. Nature 2008,454, 49-U1.

(3) Denissenko, M. F.; Chen, J. X.; Tang, M.-S.; Pfeifer, G. P.; Cytosine Methylation Determines Hot Spots of DNA Damage in the Human P53 Gene. Proc. Natl. Acad. Sci. USA 1997,94, 3893-3898.

(4) Pfeifer, G.; You, Y.; Besaratinia, A.; Mutations Induced by Ultraviolet Light. Mut. Res. 2005, 571 19-31.

(5) Tommasi, S.; Denissenko, M. F.; Pfeifer, G. P.; Sunlight Induces Pyrimidine Dimers Preferentially at 5-Methylcytosine Bases. Cancer Res. 1997,57, 4727-4730.

(6) Mitchel, D. L.; Effects of Cytosine Methylation on Pyrimidine Dimer Formation in DNA. Photochem. Photobiol. 2000,71, 162-165.

(7) Douki, T.; Cadet, J.; Individual Determination of the Yield of the Main Uv-Induced Dimeric Pyrimidine Photoproducts in DNA Suggests a High Mutagenicity of Cc Photolesions. Biochem. 2001,40, 2495-2501.

(8) Rochette, P. J.; Lacoste, S.; Therrien, J. P.; Bastien, N.; Brash, D. E.; Drouin, R.; Influence of Cytosine Methylation on Ultraviolet-Induced Cyclobutane Pyrimidine Dimer Formation in Genomic DNA. Mut. Res. 2009,665, 7-13. 
(9) Esposito, L.; Banyasz, A.; Douki, T.; Perron, M.; Markovitsi, D.; Improta, R.; Effect of C5-Methylation of Cytosine on the Photoreactivity of DNA: A Joint Experimental and Computational Study of Tcg Trinucleotides. J. Am. Chem. Soc. 2014,136, 10838-10841.

(10) You, Y. H.; Li, C.; Pfeifer, G. P.; Involvement of 5-Methylcytosine in Sunlight-Induced Mutagenesis. J. Mol. Biol. 1999,293, 493-503.

(11) Vu, B.; Cannistraro, V.; Sun, L.; Taylor, J.; DNA Synthesis Past a 5-Methylc-Containing Cis-Syn-Cyclobutane Pyrimidine Dimer by Yeast Pol Eta Is Highly Nonmutagenic Biochem. 2006,45, 9327-9335.

(12) Song, Q.; Sherrer, S. M.; Suo, Z. C.; Taylor, J. S.; Preparation of Site-Specific T = (M)Cg Cis-Syn Cyclobutane Dimer-Containing Template and Its Error-Free Bypass by Yeast and Human Polymerase Eta. J. Biol. Chem. 2012,287, 8021-8028.

(13) Markovitsi, D.; Gustavsson, T.; Vayá, I.; Fluorescence of DNA Duplexes: From Model Helices to Natural DNA. J. Phys. Chem. Lett. 2010,1, 3271-3276.

(14) McCullagh, M.; Lewis, F.; Markovitsi, D.; Douki, T.; Schatz, G. C.; Conformational Control of Tt Dimerization in DNA Conjugates. A Molecular Dynamics Study. J. Phys. Chem. B 2010,114, 5215-5221.

(15) Lee, W.; Matsika, S.; Qm/Mm Studies Reveal Pathways Leading to the Quenching of the Formation of Thymine Dimer Photoproduct by Flanking Bases. Phys. Chem. Chem. Phys. 2015,17, 9927-9935.

(16) Onidas, D.; Markovitsi, D.; Marguet, S.; Sharonov, A.; Gustavsson, T.; Fluorescence Properties of DNA Nucleosides and Nucleotides: A Refined Steady-State and Femtosecond Investigation. J. Phys. Chem. B 2002,106, 11367-11374. 
(17) Markovitsi, D.; Onidas, D.; Talbot, F.; Marguet, S.; Gustavsson, T.; Lazzarotto, E.;

Uvb/Uvc Induced Processes in Model DNA Helices Studied by Time-Resolved Spectroscopy: Pitfalls and Tricks. J. Photochem. Photobiol. A-Chem. 2006,183, 1-8.

(18) Markovitsi, D.; Gustavsson, T.; Banyasz, A., DNA Fluorescence. In Crc Handbook of Organic Photochemistry and Photobiology, Griesbeck, A.; Ghetti, F.; Oelgemoeller, M., Eds. Taylor and Francis: Boca Raton, 2012; pp 1057-1079.

(19) Lavery, R.; Zakrzewska, K.; Beveridge, D.; Bishop, T. C.; Case, D. A.; Cheatham, T.; Dixit, S.; Jayaram, B.; Lankas, F.; Laughton, C., et al.; A Systematic Molecular Dynamics Study of Nearest-Neighbor Effects on Base Pair and Base Pair Step Conformations and Fluctuations in B-DNA. Nucl. Ac. Res. 2010,38, 299-313.

(20) Pearlman, D. A.; Case, D. A.; Caldwell, J. W.; Ross, W. S.; Cheatham, T. E.; Debolt, S.; Ferguson, D.; Seibel, G.; Kollman, P.; Amber, a Package of Computer-Programs for Applying Molecular Mechanics, Normal-Mode Analysis, Molecular-Dynamics and Free-Energy Calculations to Simulate the Structural and Energetic Properties of Molecules. Comp. Phys. Comm. 1995,91, 1-41.

(21) Perez, A.; Marchan, I.; Svozil, D.; Sponer, J.; Cheatham, T. E.; Laughton, C. A.; Orozco, M.; Refinenement of the Amber Force Field for Nucleic Acids: Improving the Description of Alpha/Gamma Conformers. Biophys. J. 2007,92, 3817-3829.

(22) Cheatham, T. E.; Cieplak, P.; Kollman, P. A.; A Modified Version of the Cornell Et Al Force Field with Improved Sugar Pucker Phases and Helical Repeat. J. Biomol. Struct. Dyn. $1999,16,845-862$. 
(23) Case, D. A.; Cheatham, T. E.; Darden, T.; Gohlke, H.; Luo, R.; Merz, K. M.; Onufriev, A.; Simmerling, C.; Wang, B.; Woods, R. J.; The Amber Biomolecular Simulation Programs. J. Comput. Chem. 2005,26, 1668-1688.

(24) Berendsen, H. J. C.; Grigera, J. R.; Straatsma, T. P.; The Missing Term in Effective Pair Potentials. J. Phys. Chem. 1987,91, 6269-6271.

(25) Lankas, F.; Cheatham, T. E.; Spackova, N.; Hobza, P.; Langowski, J.; Sponer, J.; Critical Effect of the N2 Amino Group on Structure, Dynamics, and Elasticity of DNA Polypurine Tracts. Biophys. J. 2002,82, 2592-2609.

(26) Humphrey, W.; Dalke, A.; Schulten, K.; Vmd: Visual Molecular Dynamics. J. Mol. Graph. Modell. 1996,14, 33-38.

(27) Lu, X.-J.; Olson, W. K.; 3dna: A Versatile, Integrated Software System for the Analysis, Rebuilding and Visualization of Three-Dimensional Nucleic-Acid Structures. Nat. Protoc. 2008,3, 1213-1227.

(28) Lavery, R.; Moakher, M.; Maddocks, J. H.; Petkeviciute, D.; Zakrzewska, K.; Conformational Analysis of Nucleic Acids Revisited: Curves. Nucl. Ac. Res. 2009,37, 59175929.

(29) Banyasz, A.; Douki, T.; Improta, R.; Gustavsson, T.; Onidas, D.; Vayá, I.; Perron, M.; Markovitsi, D.; Electronic Excited States Responsible for Dimer Formation Upon Uv Absorption Directly by Thymine Strands: Joint Experimental and Theoretical Study. J. Am. Chem. Soc. 2012,134, 14834-14845.

(30) Sharonov, A.; Gustavsson, T.; Marguet, S.; Markovitsi, D.; Photophysical Properties of 5-Methylcytidine. Photochem. \& Photobiol. Sci. 2003,2, 362-364. 
(31) Martínez, J. M.; Elmroth, S. K. C.; Kloo, L.; Influence of Sodium Ions on the Dynamics and Structure of Single-Stranded DNA Oligomers: A Molecular Dynamics Study. J. Am. Chem. Soc. 2001,123, 12279-12289.

(32) Perez, A.; Lara Castellazzi, C.; Battistini, F.; Collinet, K.; Flores, O.; Deniz, O.; Luz Ruiz, M.; Torrents, D.; Eritja, R.; Soler-Lopez, M., et al.; Impact of Methylation on the Physical Properties of DNA. Biophys. J. 2012,102, 2140-2148.

(33) Bianchi, C.; Zangi, R.; Base-Flipping Propensities of Unmethylated, Hemimethylated, and Fully Methylated Cpg Sites. J. Phys. Chem. B 2013,117, 2348-2358.

(34) Ngo, T. T. M.; Yoo, J.; Dai, Q.; Zhang, Q.; He, C.; Aksimentiev, A.; Ha, T.; Effects of Cytosine Modifications on DNA Flexibility and Nucleosome Mechanical Stability. Nat. Commun. 2016,7.

(35) Yusufaly, T. I.; Li, Y.; Olson, W. K.; 5-Methylation of Cytosine in Cg:Cg Base-Pair Steps: A Physicochemical Mechanism for the Epigenetic Control of DNA Nanomechanics. $J$. Phys. Chem. B 2013,117, 16436-16442.

(36) Schreier, W. J.; Gilch, P.; Zinth, W.; Early Events of DNA Photodamage. Ann. Rev. Phys. Chem. 2015,66, 497-519.

(37) Markovitsi, D.; Uv-Induced DNA Damage: The Role of Electronic Excited States. Photochem. Photobiol. 2016,92, 45-51.

(38) Vayá, I.; Gustavsson, T.; Douki, T.; Berlin, Y.; Markovitsi, D.; Electronic Excitation Energy Transfer between Nucleobases of Natural DNA. J. Am. Chem. Soc. 2012,134, $11366-11368$.

(39) Chen, J.; Zhang, Y.; Kohler, B.; Excited States in DNA Strands Investigated by Ultrafast Laser Spectroscopy. Top. Curr. Chem. 2015,356, 39-87. 
(40) Plasser, F.; Aquino, A.; Lischka, H.; Nachtigallová, D.; Electronic Excitation Processes in Single-Strand and Double-Strand DNA: A Computational Approach. Top. Curr. Chem. $\mathbf{2 0 1 5}, 356,1-38$.

(41) Changenet-Barret, P.; Gustavsson, T.; Markovitsi, D.; Manet, I.; Ultrafast Electron Transfer in the Complexes of Doxorubicin with Human Telomeric G-Quadruplexes and Gc Duplexes Probed by Femtosecond Fluorescence Spectroscopy. ChemPhysChem 2016,17, 1-10. (42) Improta, R.; Photophysics and Photochemistry of Thymine Deoxy-Dinucleotide in Water: A Pcm/Td-Dft Quantum Mechanical Study. J. Phys. Chem. B 2012,116, 14261-14274.

(43) Douki, T.; Cadet, J.; Formation of Cyclobutane Dimers and (6-4) Photoproducts Upon Far-Uv Photolysis of 5-Methylcytosine-Containing Dinucleoside Monophosphates. Biochemistry 1994,33, 11942-11950.

(44) Pan, Z.; Hariharan, M.; Arkin, J. D.; Jalilov, A. S.; McCullagh, M.; Schatz, G. C.; Lewis, F. D.; Electron Donor-Acceptor Interactions with Flanking Purines Influence the Efficiency of Thymine Photodimerization (Vol 133, Pg 20793, 2011). J. Am. Chem. Soc. 2012,134, 36113611.

(45) Bouvier, B.; Dognon, J. P.; Lavery, R.; Markovitsi, D.; Millié, P.; Onidas, D.; Zakrzewska, K.; Influence of Conformational Dynamics on the Exciton States of DNA Oligomers. J. Phys. Chem. B 2003,107, 13512-13522.

(46) Ecoffet, C.; Markovitsi, D.; Millie, P.; Lemaistre, J.; Electronic Excitations in Organized Molecular Systems. A Model for Columnar Aggregates of Ionic Compounds. Chem. Phys. 1993,177, 629-643.

(47) Markovitsi, D.; Gustavsson, T.; Talbot, F.; Excited States and Energy Transfer among DNA Bases in Double Helices. Photochem. \& Photobiol. Sci. 2007,6, 717-724. 
(48) Emanuele, E.; Markovitsi, D.; Millié, P.; Zakrzewska, K.; Uv Spectra and Excitation

Delocalisation in DNA: Influence of the Spectral Width. ChemPhysChem 2005,6, 1387-1392.

(49) Hariharan, M.; McCullagh, M.; Schatz, G. C.; Lewis, F. D.; Conformational Control of Thymine Photodimerization in Single-Strand and Duplex DNA Containing Locked Nucleic Acid Tt Steps. J. Am. Chem. Soc. 2010,132, 12856-12858.

(50) Douki, T.; Meador, J. A.; Berard, I.; Wack, A.; N4-Methylation of Cytosine Drastically Favors the Formation of (6-4) Photoproducts in a Tcg Context. Photochem. Photobiol. 2015,91, $102-108$.

(51) Clivio, P.; Fourrey, J.-L.; Gasche, J.; DNA Photodamage Mechanistic Studies:

Characterization of a Thietane Intermediate in a Model Reaction Relevant to "6-4 Lesions". J. Am. Chem. Soc. 1991,113, 5481-5483.

(52) Marguet, S.; Markovitsi, D.; Time-Resolved Study of Thymine Dimer Formation. J. Am. Chem. Soc. 2005,127, 5780-5781. 
TOC Figure

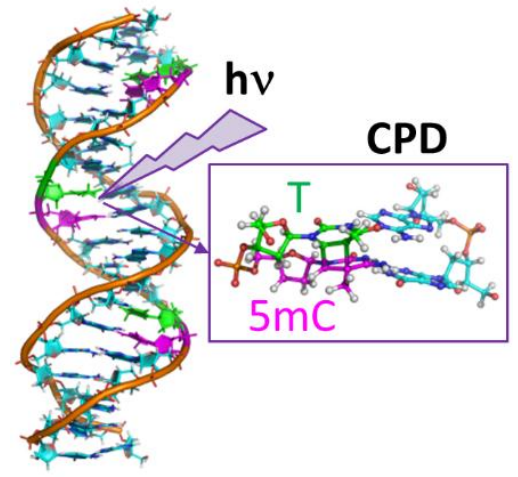

